\title{
Paper based electrochemical sensors using paper as scaffold to create porous carbon nanotube electrodes
}

\author{
Christopher J. Valentine ${ }^{1+}$, Kensuke Takagishi ${ }^{2+}$, Shinjiro Umezu ${ }^{3}$, Ronan Daly ${ }^{1 *}$, Michael De Volder ${ }^{1 *}$ \\ ${ }^{1}$ Department of Engineering, University of Cambridge, Cambridge, UK \\ ${ }^{2}$ Waseda University Graduate School of Creative Science and Engineering, Department of Modern \\ Mechanical Engineering, Tokyo, Japan \\ ${ }^{3}$ Waseda University Undergraduate School of Creative Science and Engineering, Department of \\ Modern Mechanical Engineering, Tokyo, Japan
}

Corresponding Authors: mfld2@cam.ac.uk,rd439@cam.ac.uk

Keywords: Paper based sensors, Carbon Nanotubes, Carbon Nanotubes electrodes, Porous substrates, Electrochemcial sensing, three-dimensional electrodes

\begin{abstract}
Paper-based sensors and assays have evolved rapidly due to the conversion of paper-based microfluidics, functional paper coatings, as well as new electrical and optical readout techniques. Nanomaterials have gained substantial traction as key components in paper-based sensors, as they can be coated or printed relatively easily on paper to locally control the device functionality. Here we report a new combination of methods to fabricate carbon nanotube based (CNT) electrodes for paperbased electrochemical sensors using a combination of laser cutting, drop-casting and origami. We applied this process to a range of filter papers with different porosities, and used their differences in three-dimensional cellulose networks to study the influence of the cellulose scaffold on the final CNT network and the resulting electrochemical detection of glucose. We found that an optimal porosity exists which balances the benefits of surface enhancement and electrical connectivity within the cellulose scaffold of the paper-based device and demonstrate a cost-effective process for fabrication of device arrays.
\end{abstract}

\section{Introduction}

Paper is a promising substrate for the fabrication of point-of-care in-vitro diagnostics. It is ubiquitously available, affordable, chemically stable, the unused waste is biodegradable and most importantly its porous fibrous structure provides a high surface area for reactions and drives a natural capillary-driven mass transport of sample liquids ${ }^{1-6}$. Furthermore, there are a variety of well-established technologies for manufacturing and processing of paper that can be applied to device fabrication, including folding, cutting ${ }^{7-9}$, printing ${ }^{10-13}$, masking ${ }^{14,15}$ and laser-writing ${ }^{16}$. These techniques have enabled the fabrication of paper-based microfluidic devices, where paper simultaneously acts as a channel, pump and filter ${ }^{14}$. However, paper varies enormously in terms of final composition, structure and wettability depending on the source of materials and the manufacturing process ${ }^{17}$.

The internal structure of paper is known to effect the performance of diagnostic devices ${ }^{18}$, as reported for the electrochemical sensing of nickel ${ }^{19}$, detection of $\mathrm{NADH}^{20}$ and colorimetric detection of glucose ${ }^{21}$. In the case of colorimetric detection, it was found that thicker paper types reduce the signal intensity and that papers with higher wicking speeds improve the colour development and readability of the device ${ }^{21}$. However, within the literature often little attention is paid to the paper source, which 
ranges from lab filter paper ${ }^{22,23}$ and chromatography paper ${ }^{24,25}$, to general purpose printer paper ${ }^{26}$ and paper towels ${ }^{27}$.

Electrochemical sensing is one of the most attractive opportunities for paper-based devices, especially for the detection of a variety of blood analytes, owing to its low cost, high sensitivity and high selectivity ${ }^{18}$. These sensors require high surface area electrically conductive electrodes made out of an electrochemically stable material such as a glassy carbon electrode, HOPG or other microstructured surfaces. Among these carbon nanotubes (CNTs) have been used as a transduction material in a number of applications as they have a high surface area, are electrically conductive, chemically inert and have been used as a transduction material in a variety of sensor types including resistive ${ }^{28,29}$, colorimetric ${ }^{30}$ and electrochemical sensors ${ }^{18,31}$. Furthermore, CNT powders are now regularly fabricated at an industrial scale ${ }^{32}$, and protocols have been established to make large volume suspensions ${ }^{33-35}$ that enable integration into devices. In this paper, we firstly report the role of paper porosity on the performance of CNT coated paper-based electrochemical sensors. The electrochemical sensing electrodes are fabricated by drop-casting a CNT suspension onto paper substrates of different porosities. The hypothesis is that the pore structure of the paper forms a scaffold onto which the CNTs create an electrically conductive electrode. This provides a cost-effective approach to create high surface area porous nanoparticle structures, which are at the same time resilient and easy to handle because of the paper backbone. Further, we rely on the porosity of the paper to provide micro-channels that transport the liquid sample within the electrode. Secondly, we present a novel combination of laser cutting, CNT solution drop casting and origami to manufacture arrays of diagnostic devices. Laser cutting is used here to define the electrode sensing area so that the CNT suspension can be simply drop-cast without requiring a patterning process and origami is used to connect the working, reference and counter electrode with the electrolyte (see Figure $1 \mathrm{~A}$ and process details further on).

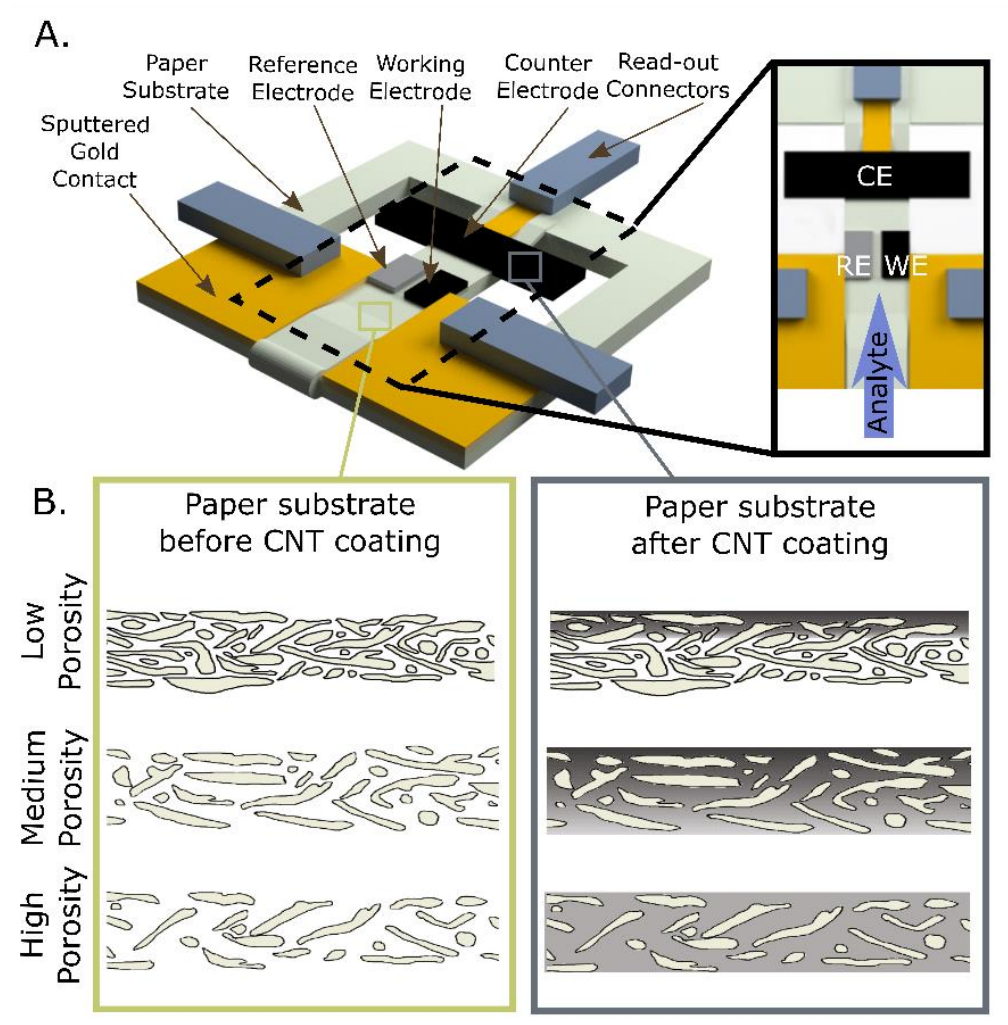


Figure 1. A) Overall architecture of the electrochemical sensors developed in this work. B) Schematic of paper porosity and the influence of the paper porosity on the CNT coating.

As sketched in Figure 1B, we compare electrodes made by coating CNT suspensions on filter papers with the same composition and thickness but with varying porosities. We hypothesise that there are several competing effects when coating the paper with CNTs. Firstly, coarse filter papers allow for a deeper penetration of CNTs during the coating process, which should improve the electrode surface area. Secondly, if the CNTs are spread too thinly through the paper due to deeper penetration, they will no longer form a percolated conductive network, which would decrease the conductivity of the coating. Finally, the mass of CNTs deposited must be sufficiently low to allow pores to remain open and allow the internal capillary flow of analyte. This suggests that a balance must be struck between the surface area and the electrical connectivity of the CNT network making up the electrode. This was verified by testing electrochemical glucose detectors as a model system representing a wider range of electrochemical diagnostic sensors. The sensor architecture developed in this paper is shown in Figure $1 \mathrm{~A}$, it consists of a typical three electrode configuration with a CNT working electrode and counter electrode and a $\mathrm{Ag} / \mathrm{AgCl}$ reference electrode. Arrays of these sensors were fabricated to study variations in performance depending on the paper porosity.

\section{Method}

The fabrication steps for the paper-based sensors are depicted in Figure 2. First, the outline of the sensor is defined using a laser cutter (Laserscript LS6040 Pro), which is used to produce an array of devices from a single sheet of filter paper (see Figure 2A). Details of the CAD file are provided in the supplementary information (SI). For ease of standardising the subsequent fabrication steps, the sensors remain attached to the paper substrate by two small strips which are torn at the end of the process to release the sensors. Here the laser is not only used to cut the sensor out of the paper, but importantly is also used to precisely define the shape of the three electrodes needed for the electrochemical sensors. By defining this geometry, the CNT suspension can simply be drop-cast onto the paper without the need for additional patterning methods (e.g. in-jet printing or screen printing) to define the electrode area. The second step of the fabrication process is to locally sputter coat a gold/palladium layer using shadow masking as shown in Figure 2B. This coating step is used to connect the sensing electrodes more easily to a potentiostat and is anticipated to be replaced with solution deposition techniques in future work.

The CNT suspension is prepared for the working and counter electrode of the sensor. The suspension is a dispersion of multi-walled CNTs (NC7000, Nanocyl, Belgium) in ethanol at a concentration of 2 $\mathrm{mg} / \mathrm{mL}$, using Nafion, at $1 \mathrm{wt} \%$, as a surfactant. The CNTs used have an average diameter of $9.5 \mathrm{~nm}$ and average length of $1.5 \mu \mathrm{m}$ according to the specification of the manufacturer. Nafion is a surfactant used for this application because of its ionic conductivity and because it enables the dispersion of CNTs in ethanol. The latter allows for faster repeated coating steps than water based dispersions ${ }^{36}$. The CNT - Nafion - ethanol suspension is sonicated for 2 hours at a $37 \mathrm{kHz}$ (Branson Bath Sonicator) and is then centrifuged for 1 hour at 5000 RCF to remove any CNT aggregates. Continuous flow processes have been developed to scale up the CNT suspension production ${ }^{33}$.

The CNT suspension is drop-cast in multiple steps onto the laser-cut electrode area to build up a range of different electrically conductive networks in the paper. This iterative process is used for both the working electrode (WE) and counter electrode (CE). For the working electrode, $0.2 \mu \mathrm{L}$ of the CNT suspension is drop-cast onto the $7.5 \mathrm{~mm}^{2}$ area in each step and for the counter electrode, $1.5 \mu \mathrm{L}$ is drop-cast onto the $1.5 \mathrm{~mm}^{2}$ area in each step. Between each deposition, the paper is dried for 2 
minutes at $60^{\circ} \mathrm{C}$. In the devices reported here, this process of drop-casting and drying is repeated twelve times for both the working and counter electrode. This amount of coating steps was chosen by measuring the evolution of the resistance of a small paper strips using the same coating process. The reference electrode (RE) is fabricated by applying $3 \mu \mathrm{L}$ of a $\mathrm{Ag} / \mathrm{AgCl}$ paste to the laser cut electrode and allowed to dry at $60^{\circ} \mathrm{C}$ for 2 minutes in the same manner as each layer of the CNT suspension on the other two electrodes.

The laser cutting process allows the accurate definition of CNT electrodes without further patterning, but because of this cutting step, there is no paper connecting the WE, CE and RE electrodes, and therefore, it is challenging to bring the analyte solution in contact with these electrodes simultaneously. This issue is solved by folding a laser cut strip of the paper back onto the three electrodes, as shown in Figure 2D. This "origami" step reconnects the electrodes, allowing the analyte to come into contact with all three electrodes during the electrochemical measurements. Finally, this entire procedure is repeated on different filter papers to study the impact of the paper porosity on the electrode performance.

The electrochemical sensors shown in Figure 2 are then characterised using a potentiostat (Biologic, VMP3) with a standard three-electrode configuration. All experiments are carried out in a saturated humid environment and at room temperature. The cyclic voltammograms (CV) are recorded using a scan rate $20.00 \mathrm{mVs}^{-1}$. Chronoamperometry (CA) measurements are conducted using an applied voltage of $0.05 \mathrm{~V}$ and a time interval of $0.1 \mathrm{~s} .5 \mu \mathrm{L}$ of $0.1 \mathrm{M}$ potassium phosphate buffer solution ( $\mathrm{pH}$ 6) was applied to the centre point of the paper device immediately before the CA measurements were run.

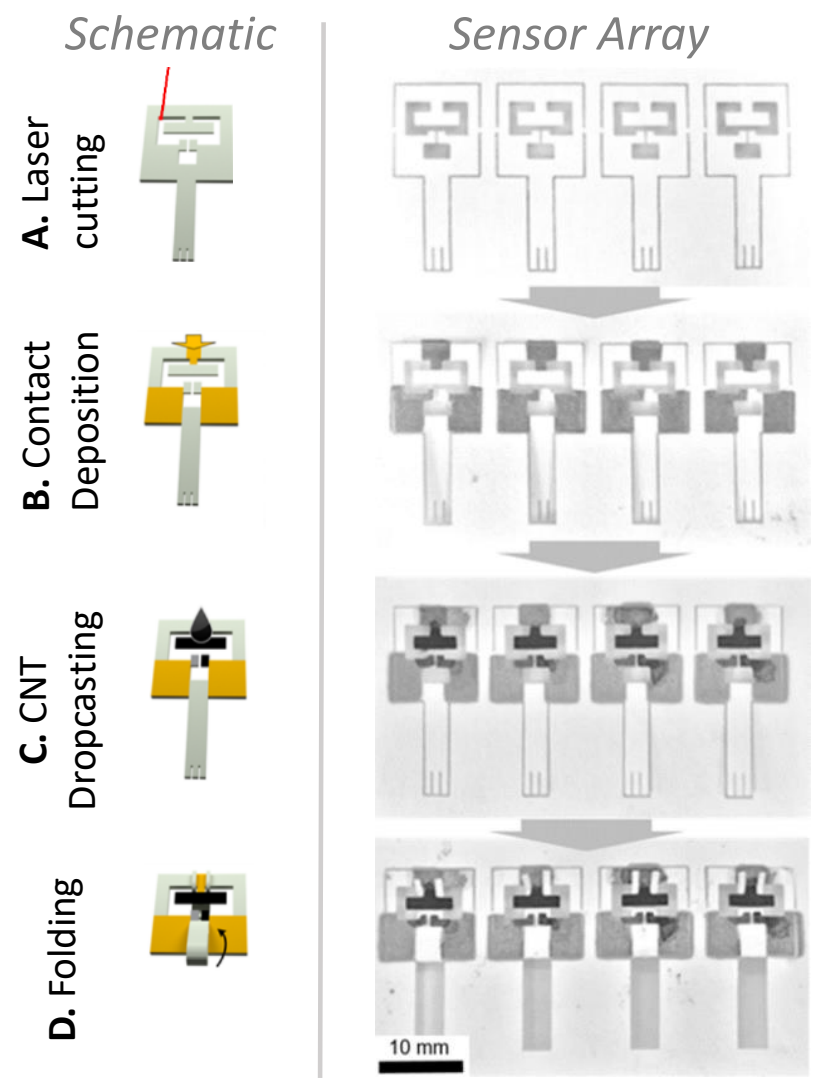

Figure 2. Images of device fabrication. The lefthand image illustrates the process occurring at each stage of fabrication. Arrays of four identical sensors are fabricated to test the repeatability of the process. A) Laser- 
cutting of sensor outline. B) Local sputtering of gold/palladium contacts using shadow masking. C) Dropcasting of suspensions on the sensor electrodes. D) Folding of paper strip onto the three electrodes.

\section{Results and Discussion}

We first compared the pore structure of the different filter papers using nitrogen physisorption (Micromeritics, 3 Flex) and analysing using Brunauer-Emmett-Teller (BET) theory to measure the surface area and Barrett-Joyner-Halenda (BJH) analysis to give a measure of the pore size distribution. The $\mathrm{BJH}$ pore distribution assumes that the pores are cylindrical in nature and that the radius of these cylinders is proportional to the sum of the Kelvin radius and the thickness of the film adsorbed ${ }^{37}$. The nitrogen physisorption isotherms used as the basis for the BET and $\mathrm{BJH}$ analysis are shown in Figures $3 \mathrm{~A}-\mathrm{C}$ for each of the filter papers with increasing porosity from $P 1$ to 3 ( $P 1$ Whatman filter paper 2; $P 2$ Whatman filter paper 1 ; and $P 3$ Whatman filter paper 4).

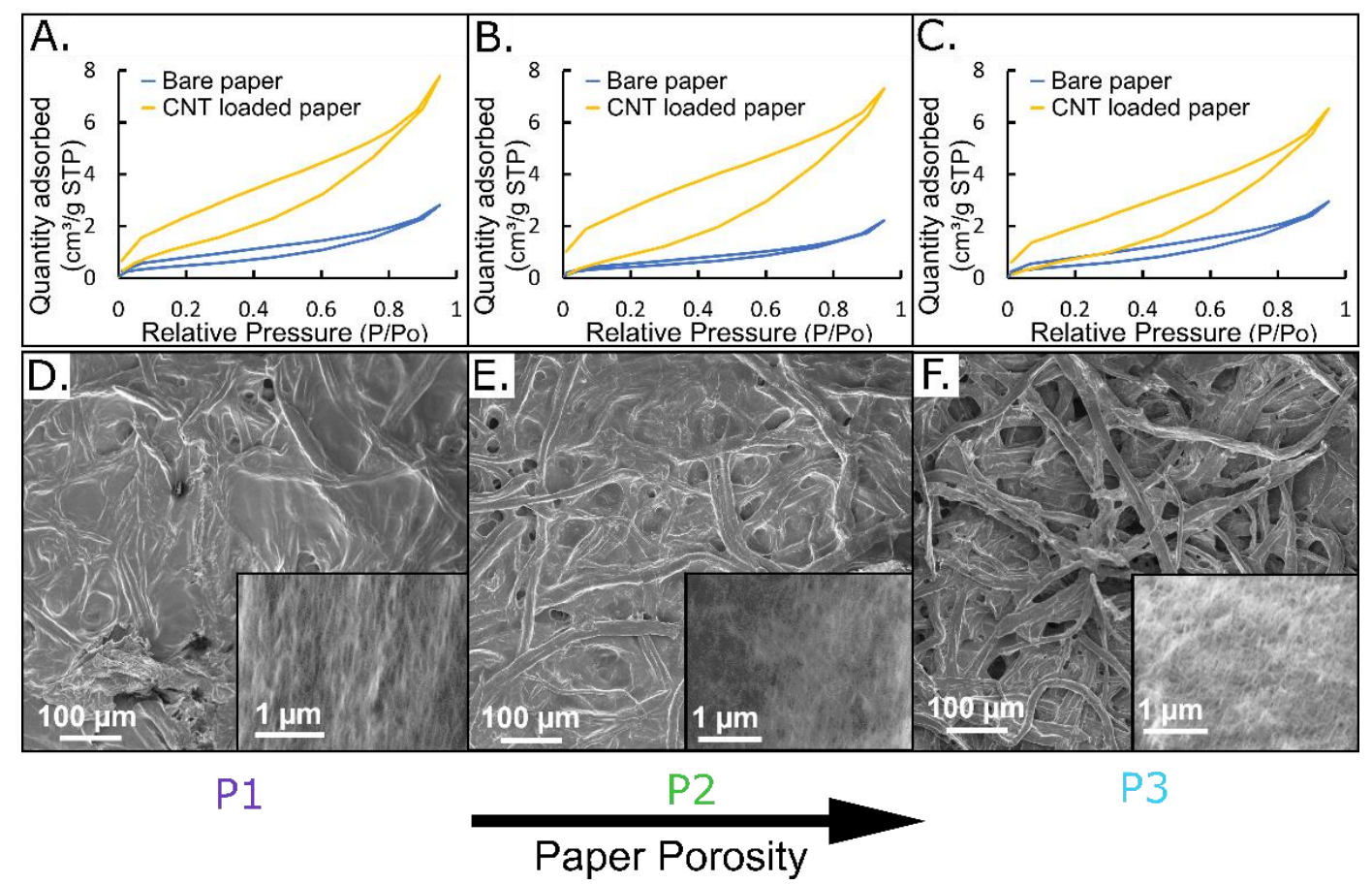

Figure 3. Analysis of the paper substrates. A) Physisorption isotherm for $P 1$ paper. B) Physisorption isotherm for $P 2$. C) Physisorption isotherm for $P 3$. D) SEM of CNT coated $P 1$ paper. E) SEM of CNT coated $P 2$ paper. F) SEM of CNT coated $P 3$ paper.

We then calculated the theoretical porosity using equation (1):

$$
\text { Porosity }=1-\frac{\rho_{\text {bulk }}}{\rho_{\text {material }}}
$$

Where $\rho_{\text {bulk }}$ is the density of the paper and $\rho_{\text {material }}$ is the nominal density of cellulose fibres (app. $\left.1.5 \times 10^{6} \mathrm{gm}^{-3},{ }^{38}\right)$. As shown in Table 1 , our calculated porosity and the manufacturer's reported nominal particle size retained suggest that $P 1$ has the smallest pore structure, followed by $P 2$, and $P 3$ has the largest pore structure. This is in agreement with SEM images shown in Figures $3 \mathrm{D}-\mathrm{F}$. Analyses from $\mathrm{BET}$ and $\mathrm{BJH}$ is give information on the increase in surface area of the electrodes as a result of CNT coating. Here a two to three-fold increase in surface area is observed after coating the cellulose with CNTs and the pore volume estimated by the BJH method shows a similar increase, as detailed in Table 
1. Close-up SEM images show the origin of this increased area with drop-casting of CNT-ethanol suspensions resulting in an even coating of the cellulose fibres with CNTs (see Figure 3D-F). We have verified that chemical composition of the three papers is similar using FT-IR (see Figure 4A) to rule out effects of surface chemistry on the coating process or device operation. The three filter papers discussed above have a similar thickness of $200 \mu \mathrm{m}$, we have however also tested a $390 \mu \mathrm{m}$ thick paper which can be found in Figure S-2 of the supporting information for comparison.

Table 1. Pore structure analysis of the filter papers used (P1, $P 2$ and $P 3)$.

\begin{tabular}{cccccccc}
$\begin{array}{c}\text { Paper } \\
\text { Type }\end{array}$ & $\begin{array}{c}\text { Porosity } \\
(\%)\end{array}$ & $\begin{array}{c}\text { Nominal } \\
\text { Particle Size } \\
\text { Retained } \\
(\mu \mathrm{m})\end{array}$ & $\begin{array}{c}\text { BET Surface } \\
\text { Area Plain } \\
\text { Paper } \\
\left(\mathrm{m}^{2} / \mathrm{g}\right)\end{array}$ & $\begin{array}{c}\text { BET Surface } \\
\text { Area CNT } \\
\& \text { Paper } \\
\left(\mathrm{m}^{2} / \mathrm{g}\right)\end{array}$ & $\begin{array}{c}\text { BET Surface } \\
\text { Area CNTs } \\
\text { only }\left(\mathrm{m}^{2} / \mathrm{g}\right)\end{array}$ & $\begin{array}{c}\text { BJH Pore } \\
\text { Volume } \\
\text { Plain Paper } \\
\left(\mathrm{mm}^{3} / \mathrm{g}\right)\end{array}$ & $\begin{array}{c}\text { BJH Pore } \\
\text { Volume CNT } \\
\text { \& Paper } \\
\left(\mathrm{mm}^{3} / \mathrm{g}\right)\end{array}$ \\
\hline P1 & 66.0 & 8 & 2.09 & 5.60 & 87.32 & 3.92 & 9.30 \\
P2 & 67.8 & 11 & 1.56 & 5.31 & 84.44 & 2.99 & 9.54 \\
P3 & 70.8 & 25 & 1.76 & 3.86 & 53.56 & 3.75 & 8.60
\end{tabular}

To test the influence of porosity on electrical conductivity with CNT coating, paper strips are laser cut out of $P 2$, and they are iteratively coated up to 20 times with $10 \mu$ of the CNT suspension described above. Figure $4 \mathrm{~B}$ shows increasing numbers of layers of CNTs (indicated by the change in colour) and the increase in electrical conductivity of a laser cut paper strip as a function of the number of CNT coating steps (see methods). Based on these results, we fabricated an electrochemical glucose sensor with the WE and CE coated with 12 layers, to balance coating time and conductivity.
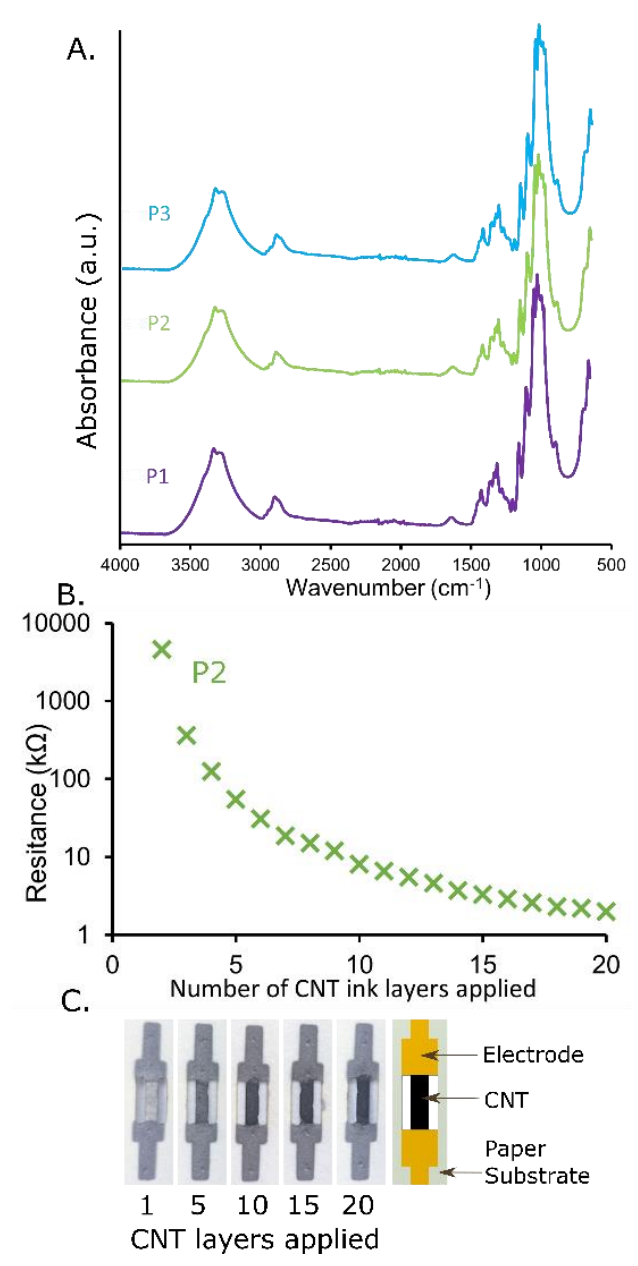
Figure 4. A) FTIR spectra of $P 1, P 2$ and $P 3$ papers. B) Resistance of CNT coated $P 2$ paper as a function of the number of suspension layers applied $\mathbf{C}$ ). Images of electrodes with increasing CNT coatings.

In Figure 5A, we report the average chronoamperometric current change as a function of the glucose concentration applied, each measurement point plotted is the mean response of three devices. As shown in Figure 5A, the current change increases with the concentration of glucose. The time response of the sensor from application of the analyte to observable response was $5-10$ seconds, and some oscillatory behaviour was observed at low glucose concentration (see Figure S-1).

Figure $5 \mathrm{~B}$, shows the current change with increasing glucose concentration. Interestingly, this measurement shows that simply changing the paper substrate can lead to a two-fold improvement in the current difference $(P 2$ versus $P 1)$. We postulate that from findings shown in Table 1 , this difference in sensitivity may be due to the CNTs forming a high surface area, with a good conducting network in $P 2$. In $P 1$ substrates, the CNTs are not expected to spread as well through the thickness of the paper resulting in a lower electrode area. Devices using $P 3$ on the other hand show a very large variation in sensitivity, which is likely due to the CNTs spreading through the paper without always forming a continuous conductive network. If as a result of this not all parts of the electrode are electrically connected, this could explain the much greater variability in device performance observed experimentally when using $P 3$ paper.

\section{A.}
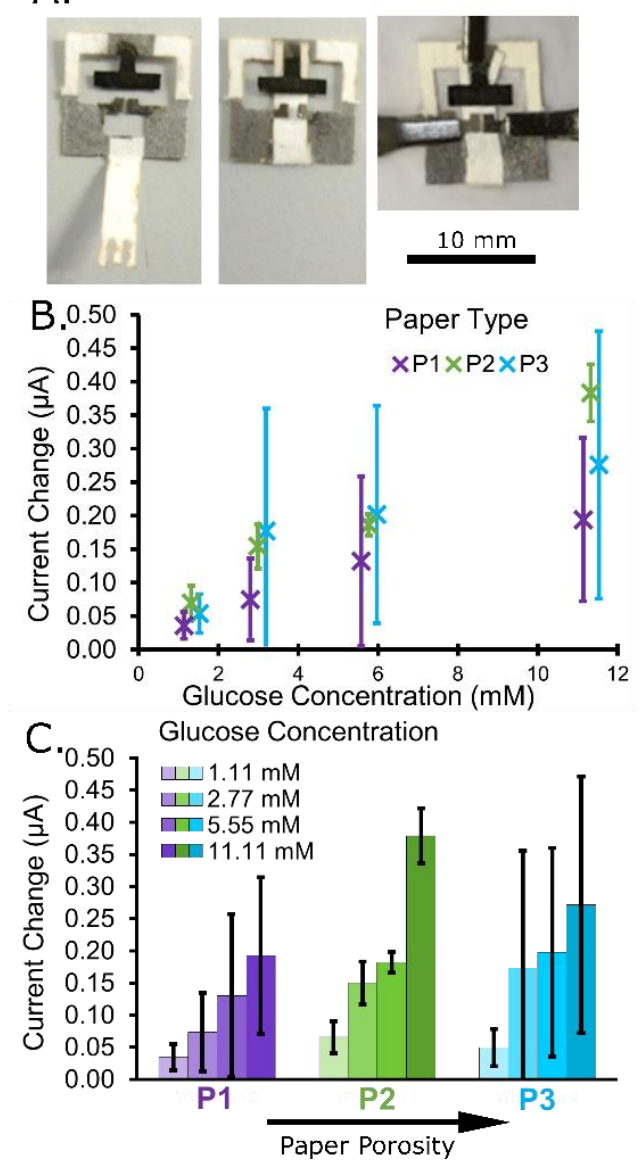

Figure 5. A) Photos showing the folding process of the devices B) The change in current as a function of glucose concentration. C) Bar chart of current change increasing with glucose concentration grouped by paper type. 
The limit of detection (LOD) was calculated as $L O D=3.3 * s / m$, where $s$ is the standard deviation of the current response of devices made from the same paper type to a known change in glucose concentration, and $\mathrm{m}$ the gradient of the linear fit to that data ${ }^{39}$. Taking the standard deviation over four devices, we found that P2 paper shows the best LOD of $2.93 \mathrm{mM}$. The normal level of glucose in whole blood is $3.5-5.3 \mathrm{mM}$ and $2.5-5.3 \mathrm{mM}$ in serum ${ }^{18}$ and so the devices fabricated from the optimal paper type would be suitable for use in the monitoring of glucose in biological samples. For the other two paper types, the LOD is found to be larger; P1 has a LOD of $3.17 \mathrm{mM}$; P3 a LOD of 8.44 $\mathrm{mM}$; showing the effect of substrate choice on the detection capabilities of the devices. Overall, these experiments demonstrate the importance of paper choice and our ability to fabricate arrays of paperbased electrochemical sensors using a new combination of laser cutting, CNT drop-casting and origami. Combined with an optimised paper porosity, this process allows for the fabrication of electrochemical sensors with comparable sensitivity and reproducibility to those reported in literature 40,41 .

\section{Summary}

Paper is an interesting substrate for the fabrication of point-of-care in-vitro diagnostic sensors because it is affordable and has a porous fibrous structure that can be used for capillary transport of liquids, for filtration and as a scaffold for coating with functional nanomaterials. In this work, we presented a new elegant fabrication process for paper-based electrochemical glucose sensors where laser cutting is used to define the area of the sensing electrodes, and origami is used to bring the analyte in contact with the electrodes. To create high surface area sensing electrodes, a CNT-Nafion suspension in ethanol is simply drop-cast on the pre-cut sensing areas. Three different types of paper substrates with increasing porosity were studied to investigate the influence of the paper porosity on the sensitivity and reproducibility of the sensor. We found that there is a trade-off between increasing paper porosity and hence surface area of the electrode and the ability of the CNTs to form a continuous electrically connected network and thus the device repeatability. Our measurements show that simply changing the porosity of the paper can lead to an almost two-fold increase in current change as well as an improvement in device-to-device reproducibility. With the growing interest in low-cost paper-based sensor development, this model sensor is useful to demonstrate the importance of paper choice and an understanding of the behaviour of functional coatings within the cellulose fibre matrix, to ensure reliable and repeatable device fabrication.

\section{Acknowledgements}

The authors would like to acknowledge $\mathrm{C}$. George for his advice on the electrochemical measurements made, N. Chiodarelli for his advice about the formulation of the CNT suspensions and Y. Shams for her advice on the laser cutting of paper. The Engineering and Physical Sciences Research Council [EP/L015889/1] and the ERC Starting Grant 337739-HIENA supported this work

\section{Supporting Information}

The supporting information contains computer aided drawing of laser cutter outline and figures of fourth thicker paper type.

\section{References:}

(1) Anovitz, L. M.; Cole, D. R. Characterization and Analysis of Porosity and Pore Structures. Rev. Mineral. Geochemistry 2015, 80 (1), 61-164. https://doi.org/10.2138/rmg.2015.80.04. 
(2) Akram, M. S.; Daly, R.; da Cruz Vasconcellos, F.; Yetisen, A. K.; Hutchings, I.; Hall, E. A. H. Applications of Paper-Based Diagnostics. In Lab-on-a-Chip Devices and Micro-Total Analysis Systems; Springer International Publishing: Cham, 2015; pp 161-195.

https://doi.org/10.1007/978-3-319-08687-3_7.

(3) Yang, Y.; Noviana, E.; Nguyen, M. P.; Geiss, B. J.; Dandy, D. S.; Henry, C. S. Paper-Based Microfluidic Devices: Emerging Themes and Applications. Anal. Chem. 2017, 89 (1), 71-91. https://doi.org/10.1021/acs.analchem.6b04581.

(4) Zhang, Y.; Zhang, L.; Cui, K.; Ge, S.; Cheng, X.; Yan, M.; Yu, J.; Liu, H. Flexible Electronics Based on Micro/Nanostructured Paper. Adv. Mater. 2018, 30 (51), 1801588.

https://doi.org/10.1002/adma.201801588.

(5) Zhang, J.; Bokov, A. A.; Gao, S. L.; Zhang, N.; Zhuang, J.; Ren, W.; Ye, Z. G. Effect of Hierarchical Structure on Electrical Properties and Percolation Behavior of Multiscale Composites Modified by Carbon Nanotube Coating. Compos. Sci. Technol. 2018, 164 (May), 160-167. https://doi.org/10.1016/j.compscitech.2018.05.037.

(6) Li, Z.; Liu, H.; He, X.; Xu, F.; Li, F. Pen-on-Paper Strategies for Point-of-Care Testing of Human Health. TrAC - Trends Anal. Chem. 2018, 108, 50-64.

https://doi.org/10.1016/j.trac.2018.08.010.

(7) Fenton, E. M.; Mascarenas, M. R.; López, G. P.; Sibbett, S. S. Multiplex Lateral-Flow Test Strips Fabricated by Two-Dimensional Shaping. ACS Appl. Mater. Interfaces 2009, 1 (1), 124-129. https://doi.org/10.1021/am800043z.

(8) Nie, J.; Liang, Y.; Zhang, Y.; Le, S.; Li, D.; Zhang, S. One-Step Patterning of Hollow Microstructures in Paper by Laser Cutting to Create Microfluidic Analytical Devices. Analyst 2013, 138 (2), 671-676. https://doi.org/10.1039/C2AN36219H.

(9) Spicar-Mihalic, P.; Toley, B.; Houghtaling, J.; Liang, T.; Yager, P.; Fu, E. CO 2 Laser Cutting and Ablative Etching for the Fabrication of Paper-Based Devices. J. Micromechanics Microengineering 2013, 23 (6), 067003. https://doi.org/10.1088/0960-1317/23/6/067003.

(10) Li, X.; Tian, J.; Garnier, G.; Shen, W. Fabrication of Paper-Based Microfluidic Sensors by Printing. Colloids Surfaces B Biointerfaces 2010, 76 (2), 564-570. https://doi.org/10.1016/j.colsurfb.2009.12.023.

(11) Nie, Z.; Nijhuis, C. A.; Gong, J.; Chen, X.; Kumachev, A.; Martinez, A. W.; Narovlyansky, M.; Whitesides, G. M. Electrochemical Sensing in Paper-Based Microfluidic Devices. Lab Chip 2010, 10 (4), 477-483. https://doi.org/10.1039/B917150A.

(12) Metters, J. P.; Gomez-Mingot, M.; Iniesta, J.; Kadara, R. O.; Banks, C. E. The Fabrication of Novel Screen Printed Single-Walled Carbon Nanotube Electrodes: Electroanalytical Applications. Sensors Actuators B Chem. 2013, 177, 1043-1052. https://doi.org/10.1016/j.snb.2012.11.078.

(13) Carrilho, E.; Martinez, A. W.; Whitesides, G. M. Understanding Wax Printing: A Simple Micropatterning Process for Paper-Based Microfluidics. Anal. Chem. 2009, 81 (16), 70917095. https://doi.org/10.1021/ac901071p.

(14) Martinez, A. W.; Phillips, S. T.; Butte, M. J.; Whitesides, G. M. Patterned Paper as a Platform for Inexpensive, Low-Volume, Portable Bioassays. Angew. Chemie Int. Ed. 2007, 46 (8), 13181320. https://doi.org/10.1002/anie.200603817.

(15) Songjaroen, T.; Dungchai, W.; Chailapakul, O.; Laiwattanapaisal, W. Novel, Simple and LowCost Alternative Method for Fabrication of Paper-Based Microfluidics by Wax Dipping. Talanta 2011, 85 (5), 2587-2593. https://doi.org/10.1016/j.talanta.2011.08.024.

(16) Zang, X.; Shen, C.; Chu, Y.; Li, B.; Wei, M.; Zhong, J.; Sanghadasa, M.; Lin, L. Laser-Induced 
Molybdenum Carbide-Graphene Composites for 3D Foldable Paper Electronics. Adv. Mater. 2018, 30 (26), 1800062. https://doi.org/10.1002/adma.201800062.

(17) Lappalainen. Cellulose as a Novel Substrate for Lateral Flow Assay. Nord. Pulp Pap. Res. J. 2010, 25 (04), 536-550. https://doi.org/10.3183/NPPRJ-2010-25-04-p536-550.

(18) Dungchai, W.; Chailapakul, O.; Henry, C. S. Electrochemical Detection for Paper-Based Microfluidics. Anal. Chem. 2009, 81 (14), 5821-5826. https://doi.org/10.1021/ac9007573.

(19) Nurak, T.; Praphairaksit, N.; Chailapakul, O. Fabrication of Paper-Based Devices by Lacquer Spraying Method for the Determination of Nickel (II) lon in Waste Water. Talanta 2013, 114, 291-296. https://doi.org/10.1016/j.talanta.2013.05.037.

(20) Metters, J. P.; Houssein, S. M.; Kampouris, D. K.; Banks, C. E. Paper-Based Electroanalytical Sensing Platforms. Anal. Methods 2013, 5 (1), 103-110. https://doi.org/10.1039/C2AY26396C.

(21) Evans, E.; Gabriel, E. F. M.; Coltro, W. K. T.; Garcia, C. D. Rational Selection of Substrates to Improve Color Intensity and Uniformity on Microfluidic Paper-Based Analytical Devices. Analyst 2014, 139 (9), 2127-2132. https://doi.org/10.1039/C4AN00230J.

(22) Nery, E. W.; Kubota, L. T. Sensing Approaches on Paper-Based Devices: A Review. Anal. Bioanal. Chem. 2013, 405 (24), 7573-7595. https://doi.org/10.1007/s00216-013-6911-4.

(23) Liana, D. D.; Raguse, B.; Gooding, J. J.; Chow, E. Recent Advances in Paper-Based Sensors. Sensors 2012, 12 (9), 11505-11526. https://doi.org/10.3390/s120911505.

(24) Nie, Z.; Deiss, F.; Liu, X.; Akbulut, O.; Whitesides, G. M. Integration of Paper-Based Microfluidic Devices with Commercial Electrochemical Readers. Lab Chip 2010, 10 (22), 3163. https://doi.org/10.1039/c0lc00237b.

(25) Bracher, P. J.; Gupta, M.; Mack, E. T.; Whitesides, G. M. Heterogeneous Films of Ionotropic Hydrogels Fabricated from Delivery Templates of Patterned Paper. ACS Appl. Mater. Interfaces 2009, 1 (8), 1807-1812. https://doi.org/10.1021/am900340m.

(26) Saha, B.; Baek, S.; Lee, J. Highly Sensitive Bendable and Foldable Paper Sensors Based on Reduced Graphene Oxide. ACS Appl. Mater. Interfaces 2017, 9 (5), 4658-4666. https://doi.org/10.1021/acsami.6b10484.

(27) Martinez, A. W.; Phillips, S. T.; Whitesides, G. M.; Carrilho, E. Diagnostics for the Developing World: Microfluidic Paper-Based Analytical Devices. Anal. Chem. 2010, 82 (1), 3-10. https://doi.org/10.1021/ac9013989.

(28) Han, J.-W.; Kim, B.; Li, J.; Meyyappan, M. Carbon Nanotube Based Humidity Sensor on Cellulose Paper. J. Phys. Chem. C 2012, 116 (41), 22094-22097. https://doi.org/10.1021/jp3080223.

(29) De Volder, M.; Tawfick, S. H.; Copic, D.; Hart, A. J. Hydrogel-Driven Carbon Nanotube Microtransducers. Soft Matter 2011, 7 (21), 9844. https://doi.org/10.1039/c1sm06215h.

(30) Rattanarat, P.; Dungchai, W.; Cate, D.; Volckens, J.; Chailapakul, O.; Henry, C. S. Multilayer Paper-Based Device for Colorimetric and Electrochemical Quantification of Metals. Anal. Chem. 2014, 86 (7), 3555-3562. https://doi.org/10.1021/ac5000224.

(31) Lv, Y.; Jin, S.; Wang, Y.; Lun, Z.; Xia, C. Recent Advances in the Application of Nanomaterials in Enzymatic Glucose Sensors. J. Iran. Chem. Soc. 2016, 13 (10), 1767-1776. https://doi.org/10.1007/s13738-016-0894-y.

(32) De Volder, M. F. L.; Tawfick, S. H.; Baughman, R. H.; Hart, A. J. Carbon Nanotubes: Present and Future Commercial Applications. Science (80-. ). 2013, 339 (6119), 535-539. https://doi.org/10.1126/science.1222453. 
(33) Chiodarelli, N.; De Volder, M. High-Throughput and Consistent Production of Aqueous Suspensions of Single-Wall Carbon Nanotubes. Carbon N. Y. 2019, 145, 757-763. https://doi.org/10.1016/j.carbon.2019.01.026.

(34) Backes, C.; Szydłowska, B. M.; Harvey, A.; Yuan, S.; Vega-Mayoral, V.; Davies, B. R.; Zhao, P.; Hanlon, D.; Santos, E. J. G.; Katsnelson, M. I.; Blau, W. J.; Gadermaier, C.; Coleman, J. N. Production of Highly Monolayer Enriched Dispersions of Liquid-Exfoliated Nanosheets by Liquid Cascade Centrifugation. ACS Nano 2016, 10 (1), 1589-1601. https://doi.org/10.1021/acsnano.5b07228.

(35) Yoon, H.; Yamashita, M.; Ata, S.; Futaba, D. N.; Yamada, T.; Hata, K. Controlling Exfoliation in Order to Minimize Damage during Dispersion of Long SWCNTs for Advanced Composites. Sci. Rep. 2015, 4 (1), 3907. https://doi.org/10.1038/srep03907.

(36) Wang, J.; Musameh, M.; Lin, Y. Solubilization of Carbon Nanotubes by Nafion toward the Preparation of Amperometric Biosensors. J. Am. Chem. Soc. 2003, 125 (9), 2408-2409. https://doi.org/10.1021/ja028951v.

(37) Joyner, L. G.; Barrett, E. P.; Skold, R. The Determination of Pore Volume and Area Distributions in Porous Substances. II. Comparison between Nitrogen Isotherm and Mercury Porosimeter Methods. J. Am. Chem. Soc. 1951, 73 (7), 3155-3158. https://doi.org/10.1021/ja01151a046.

(38) Adusumali, R.-B.; Reifferscheid, M.; Weber, H.; Roeder, T.; Sixta, H.; Gindl, W. Mechanical Properties of Regenerated Cellulose Fibres for Composites. Macromol. Symp. 2006, 244 (1), 119-125. https://doi.org/10.1002/masy.200651211.

(39) Long, G. L.; Winefordner, J. D. Limit of Detection. A Closer Look at the IUPAC Definition. Anal. Chem. 1983, 55 (7), 712A-724A. https://doi.org/10.1021/ac00258a001.

(40) Qin, H.; Zhu, Z.; Ji, W.; Zhang, M. Carbon Nanotube Paper-Based Electrode for Electrochemical Detection of Chemicals in Rat Microdialysate. Electroanalysis 2018, 30, $1022-$ 1027. https://doi.org/10.1002/elan.201700689.

(41) Guadarrama-Fernández, L.; Novell, M.; Blondeau, P.; Andrade, F. J. A Disposable, Simple, Fast and Low-Cost Paper-Based Biosensor and Its Application to the Determination of Glucose in Commercial Orange Juices. Food Chem. 2018, 265 (May), 64-69.

https://doi.org/10.1016/j.foodchem.2018.05.082. 
